\title{
Creating advocates for mammal conservation through citizen science
}

\author{
Tavis D. Forrester ${ }^{*, a, 1}$, Megan Baker ${ }^{\mathrm{a}, 2}$, Robert Costello ${ }^{\mathrm{b}}$, Roland Kays ${ }^{\mathrm{b}, \mathrm{c}, \mathrm{d}}$, Arielle W. Parsons ${ }^{\mathrm{c}}$, William J. \\ McShea $^{\mathrm{a}}$
}

\begin{abstract}
Citizen science initiatives have shown promise to provide informal education about nature and conservation and simultaneously gather scientific data at large scales. eMammal is a platform for citizen science projects that recruits volunteers to place camera traps that collect data in the form of wildlife photographs. Our project offered informal education on wildlife ecology and conservation to volunteers through training materials, feedback during the project, and a natural history blog. We tested whether our education efforts and volunteer activities affected their project specific skills, wildlife knowledge, conservation attitudes, and what kind of information they shared with their social network. Volunteers accurately $(>90 \%)$ identified 15 of 20 wildlife species captured in the photos and reduced the rejection rate of camera placements over time. Our surveys showed that volunteer's attitudes toward conservation were high before joining the project and did not change after participating. However, volunteer knowledge of wildlife was higher after working with eMammal. Volunteers also became advocates for mammal conservation by sharing their new knowledge. Roughly half of our volunteers reported actively discussing some type of information related to wildlife both before (50\%) and after (54\%) the project. However, after volunteering they were $84 \%$ more likely to discuss local mammals or local mammal conservation. The likelihood of discussing local mammals was positively influenced by the number of predator photos captured by volunteers, showing that the type of experience can influence how information is spread through a volunteer's social network. Citizen science can connect people to the natural world while simultaneously providing reliable data for conservation.
\end{abstract}

Keywords

Camera trap; conservation attitudes; informal education; social network; wildlife 
Solving conservation problems requires both scientific data and public support for conservation actions, and generating this support often requires broad engagement with the public (Opdam \& Wascher 2004; Cooper et al. 2007; Pressey et al. 2007). Informal education is an important avenue for engaging the public with both science and nature, and often takes place in natural history museums, zoos or aquariums, which a majority of American adults report visiting (Falk \& Dierking 2010). Informal learning is an important avenue for science education among adults (Falk \& Dierking 2010) and is also a strategy for maintaining support for conservation (Ballard et al. In Press; Ballantyne et al. 2007).

Citizen science, engaging non-professional volunteers in scientific research (Bonney et al. 2009; Tulloch et al. 2013), has been advanced as a method to collect ecological data at large-scales (Miller-Rushing et al. 2012) as well as an effective form of informal education about the natural world (Cooper et al. 2007; Dickinson et al. 2012). There is still some question whether citizen science projects can achieve simultaneous goals of data collection and education (Jordan et al. 2011), although several projects have combined data collection with informal education (Ballard et al. In press; Jordan et al. 2011). Understanding volunteer motivations and establishing explicit education goals can improve informal education outcomes (Domroese \& Johnson In press), but there is evidence that even without explicit learning goals, volunteers can learn about ecology and science simply through the experience of rigorous data collection (Ballard et al. In Press; Jordan et al. 2009). Projects that are collecting data for scientific research offer experience with "authentic science" that provides unique informal education opportunities (Jordan et al. 2012b) that can be easily supplemented with additional educational activities.

Citizen science is unique in that in can provide a vehicle to directly experience the natural world as well as offering informal education. Direct experience with nature may be a useful tool to encourage support for conservation. As the world has become increasingly urbanized (Alig et al. 2004; Cohen 2006) the public in industrialized countries has spent less time engaged in nature-based recreation (Pergams \& Zaradic 2006, 2008). Children, and particularly urban children, are spending much less time outdoors than previous generations (Louv 2005). This trend of reduced interaction with the natural world may negatively impact current and future support for conservation (Miller 2005), and there is already evidence that the decline in some types of outdoor recreation is impacting support for non-governmental organizations that are focused on conservation (Zaradic et al. 2009). Citizen science offers one strategy to combat this trend and provide meaningful interactions with the natural world as well give volunteers experience with science, and often science that is focused on conservation.

To evaluate the possible effects of citizen science on support for conservation, we investigated the effects of volunteering for a large wildlife ecology project. We evaluated volunteer experience, learning outcomes, and possible changes to conservation attitudes before and after a wildlife study on the effects of recreation on mammals using eMammal. eMammal is a platform for gathering, storing, and sharing survey data of mammals collected with camera traps (McShea et al. 2016). Camera traps are motiontriggered cameras that photograph any animal larger than $\sim 100 \mathrm{~g}$ that moves through the sensor field. The goals of eMammal are to provide tools for large-scale camera trapping for researchers and citizen scientists and to maintain a data repository of mammal survey data that can be easily and quickly accessed for conservation and management. We assessed the quality of volunteer data and how quickly volunteers learned to place cameras and identify wildlife pictures through expert review of all photos identified by volunteers. The effectiveness of project activities in changing volunteer attitudes, knowledge and behavior were assessed with pre and post-season surveys of volunteers and control groups. We predicted that volunteers would: 1) improve their ability to setup cameras and identify species over time; 2) increase their knowledge about the natural history of mammals; 3 ) improve attitudes towards 
conservation; 4) share information learned during or from the project with more people in their networks, and 5) be more likely to share information if they detected rare or charismatic wildlife, such as predators, on their camera traps.

\section{Materials and methods}

\section{1 eMammal project description}

eMammal was developed by the Smithsonian Institution and the North Carolina Museum of Natural Sciences (McShea et al. 2016). Program features include online tools for managing volunteers, custom applications for identifying wildlife photographs, uploading data, and reviewing volunteer identifications, and storage of data in the Smithsonian Data Repository. The data reported here are from a study to evaluate the effect of recreation on wildlife across 32 public lands and six states in the mid-Atlantic region of the USA (Figure 1). Volunteers were recruited to deploy cameras at pre-assigned locations determined by project scientists. Volunteers were recruited for three different survey efforts; the summer and fall of 2012, the spring and summer of 2013, and the summer and fall of 2013. Each survey effort was three to four months long. Volunteers placed 3-6 cameras every three weeks and identified and uploaded wildlife photos captured on their cameras using eMammal software tools.

2.2 Volunteer recruitment, training, and feedback Virginia, South Carolina and Tennessee Master Naturalists, the Sierra Club, Appalachian Trail Clubs, 4$\mathrm{H}$, local colleges and universities, and various "Friends Groups" associated with partners or parks. All volunteers attended a $\sim 4 \mathrm{hr}$ training session where they learned to use wildlife cameras as well as the eMammal website and software. Trainings included a physical demonstration of how to setup and deploy wildlife cameras and a hands-on practice session where the volunteers received feedback from project staff.

After the training volunteers were assigned camera locations, placed their cameras, collected the photos and relocated the cameras after three weeks, and recorded site-specific data. They used the eMammal software to identify the species captured and upload photographs and metadata to a cloud database (McShea et al. 2016). At the expert review website project staff reviewed camera setup, rejected cameras that were set improperly (e.g. too high, too low, obstructed view) and confirmed all species identifications. During 2013 eMammal staff contacted many volunteers when their cameras had been rejected and explained why.

Educational efforts were conducted in person and online. Project scientists discussed the questions motivating the eMammal project as part of the initial in-person training, and during the 2013 season project staff also wrote articles for the eMammal blog about the natural history of mammals in the midAtlantic. At the end of each season volunteers were invited to attend a gathering where project staff summarized the results of that survey season.

Volunteers received three difference kinds of feedback from project staff. Volunteers received direct feedback on camera setup and any questions at the initial training, eMammal staff contacted volunteers directly to discuss why camera setups had been rejected in 2013, and volunteers received emails reminding to read the eMammal blog. Volunteers also used training materials to give self-feedback about camera setup and information found online to learn about mammals.

2.3 Volunteer project specific skills and data quality 
We measured data quality of volunteers by comparing the species identifications of experts to that of the volunteers (McShea et al. 2016). We measured volunteer learning of project skills by comparing the accuracy of species identification and camera rejection rates over the first 3 months they volunteered. We measured improvement in species identification by comparing the species identification of two pairs of common and visually similar species that had volunteer identification rates below $90 \%$ (red fox (Vulpes vulpes) vs. gray fox (Urocyon cinereoargenteus); fox squirrel (Sciurus niger) vs. eastern gray squirrel (Sciurus carolinensis)).

\subsection{Volunteer knowledge and attitudes toward conservation}

All survey efforts were approved by the Smithsonian's Institutional Review Board (IRB protocol HS12047). We used a pre-test/post-test, control/treatment approach to surveying volunteer project related knowledge and conservation attitudes (Campbell \& Stanley 1963). We surveyed all volunteers immediately after their initial training and after participation in the project, and we also administered the pre and post-surveys to a control group of non-volunteers. The control group was recruited from the same community groups as our volunteers, and we surveyed a separate control and volunteer group in each survey season (summer 2012, spring/summer 2013, and summer/fall 2013). Both groups were surveyed with an online survey composed of six sections, plus a section requesting basic demographic information. The first section was a quiz of wildlife species in camera trap photos and the second section was a quiz that assessed their knowledge of the natural history of mammals. The third, fourth, and fifth sections were questions about their attitudes towards wildlife, protected areas, and the interface of conservation and economics respectively. The sixth section was questions about whether volunteers had recently talked to anyone about wildlife and what they had discussed (see Appendix A for sample survey). The survey included modified versions of previously published survey instruments and questions created by project staff (see below for details).

To assess mammal identification we asked volunteers to identify animal species in camera trap photographs by picking from the species listed in the eMammal desktop application. As in the desktop app, there was no help text, volunteers made their best choice from the available list. We measured volunteer knowledge of the natural history of wildlife with 10 multiple choice questions focused on the natural history (e.g. foraging behavior) of species commonly encountered in camera trap surveys in the mid-Atlantic. Questions were created by project researchers and reviewed by Smithsonian education staff. Natural history questions were analyzed separately for 2012 and the two 2013 seasons because 2012 natural history questions were true-false and both 2013 surveys used multiple choice questions.

We used a subset of the Wildlife Attitudes and Values Scale (WAVS) to gauge volunteer attitudes toward wildlife (Purdy \& Decker 1989). The WAVS instrument was created to measure how people valued wildlife and contains three main groups of attitudes: traditional-conservation, societal-benefits, and problem-acceptance (Purdy \& Decker 1989). We chose at least one question from each major group for a total of five questions. We used questions about the balance between economics and conservation and the status of natural areas in the region from the Conservation in the West survey (Weigel \& Metz 2014). This an existing annual survey on conservation attitudes that has been conducted across five western states for the last 6 years and validated and tested for bi-partisan audiences.

eMammal staff created questions about the contribution of local, regional, and national natural areas to their quality of life. We asked volunteers to rank the contribution of each type of area to their quality of life on a Likert scale ranging from 'not important' to 'very important' with a 'no opinion' option. Finally, to measure how often volunteers shared information about wildlife we asked volunteers to self-report if they had recently discussed wildlife or wildlife-related issues. Follow-up questions were asked if they 
indicated they had a discussion within the last 2 months, including the topic of their most recent discussion, and if they had taught some wildlife concept during the interaction.

All questions were face validated by project scientists and were content validated with a test survey that gave respondents the opportunity to give feedback on each survey page. A group of Smithsonian volunteers not associated with eMammal took a draft version of the survey and gave feedback on survey clarity and ease of navigation (Dillman 2011).

We assessed volunteer experience by asking them to describe the best and the worst aspect of volunteering for the project at the end of the season. We also surveyed a subset of volunteers before they worked on the project and asked them to rank what they expected to be the most rewarding part of the project from a list of project components. We asked them the same question at the end of the survey season.

We collected standard demographic information including year of birth, gender, zip code, household income, education level, and formal education in wildlife or environmental sciences. We classified household income into 4 broad categories ( $>\$ 20 \mathrm{k}, \$ 20-\$ 60 \mathrm{k}, \$ 60-\$ 100 \mathrm{k},>\$ 100 \mathrm{k})$, education level into three categories (High School, College, Professional/Graduate Degree), and wildlife education into three self-assessed groups (None, Some, A lot).

\subsection{Analysis}

We calculated the accuracy of species identification by dividing volunteer correct identifications by expert identifications for each of the 15 most commonly detected species. We tested change in the accuracy of species ID and percent of rejected camera deployments over the first 3 months that volunteers participated using a repeated-measures ANOVA or a Friedman test if the response variable did not meet assumptions of normality.

We compared the pre and post-test scores on the species identification and natural history tests of both groups using paired $t$-tests or Wilcoxon signed rank tests if the scores did not meet assumptions of normality and could not be transformed. We also conducted an experiment on the effectiveness of the eMammal blog as an outreach tool during the fall of 2013. The control and volunteer groups were randomly divided in half, and one half of each group was regularly invited to read blog posts on natural history of wildlife written by lead author (T. Forrester), and we compared the change in natural history scores between the blog and control groups. During the post-season survey both volunteer and control respondents were asked if they had read the eMammal blog. We used this question to measure how many of the treatment group actually read the blog and if any of the control group had found the blog and read the articles.

We scored Likert scale questions on a 1-5 scale, with some items reverse coded as appropriate. We generated indices for attitudes toward wildlife, natural areas, and conservation economics by summing the responses to several Likert scale questions. Paired $t$-tests were used to test for differences in the pre and post-test values, or Wilcoxon rank tests were used if the indices did not meet assumptions of normality and could not be transformed.

We compared the difference in rates of self-reported discussions of mammals and mammal conservation between our volunteers and the control group using McNemar's test in a case-control framework (Eliasziw \& Donner 1991). We scored what the volunteer discussed during their most recent wildlife communications, and used McNemar's test to measure the difference between how often volunteers shared information about local mammals and their conservation before and after volunteering. All reports of discussing mammals native to the mid-Atlantic region, or conservation issues affecting those 
mammals, were scored as a one, while discussion of other mammals (i.e. polar bears), other wildlife, or non-local conservation issues were scored as a zero.

We also conducted a post-hoc analysis of factors that influenced the likelihood of volunteers sharing information about local mammals and their conservation. We used a generalized linear model with a logit link to assess how demographic factors, the number of wildlife detections, and the number of only predator detections uploaded by each volunteer affected the likelihood that volunteers would report discussing local mammals or conservation. We hypothesized that education level, income, gender, age, and the location and housing density (rural, town/suburb, or city) where the volunteer lived would affect the likelihood of information sharing. Variables were evaluated for collinearity $\left(r^{2}>0.50\right.$ or significant chisquare result) and for those variables we selected the variable that had more complete responses or had the best model fit in single variable logit models. We determined the volunteer residency by obtaining the latitude and longitude of the volunteer's zip code. The longitude, latitude, and latitude*longitude were tested in single models and we included the best performing covariate in our model set. We compared a model set of all subsets of demographic/spatial variables and all detections of predators to a model set including all subsets of personal variables and all wildlife detections. We selected the top models based on $\mathrm{AIC}$ values corrected for small sample sizes $\left(\mathrm{AIC}_{\mathrm{c}}\right.$ ) and considered a difference of $>2$ in $\mathrm{AIC}_{\mathrm{c}}$ score to indicate a better model (Burnham \& Anderson 2002). We considered nested models within $\triangle 2 \mathrm{AIC}_{\mathrm{c}}$ of the best model as competitors for best model only if parameters were not merely a subset of the best model and parameters in the model were significant at the 0.1 level (Burnham \& Anderson 2002; Arnold 2010). If multiple non-nested models were within $\Delta 2 \mathrm{AIC}_{\mathrm{c}}$ of the best model we report model-averaged parameter estimates (Burnham \& Anderson 2002).

\section{Results}

\subsection{Data accuracy and camera deployment quality}

Volunteers processed 183,850 sequences of wildlife, humans, and blank photos that included over 2.1 million individual photos, and identified 10 of the 15 most common wildlife species with accuracy rates of over $90 \%$ (McShea et al. 2016). Volunteers had lower accuracy with two closely related squirrels, two fox species, and low quality images (Table 1). Volunteers showed no improvement in the identification of these species over three months of project participation (Friedman test, $\chi_{112}^{2}=1.02, \mathrm{df}=2, p=0.59$; Table 1). Volunteers classified $2.7 \%$ of the wildlife detections as 'unknown animal', but wildlife experts were able to identify animals to genus or species in $61 \%$ of these detections. Experts rejected $5 \%$ of all camera deployments because of improper setup, and another $1.7 \%$ for equipment failure. Volunteers improved their camera set-up over time and showed a significant reduction in rejection rates for each month they spent volunteering for the project (Friedman test, $\chi_{70}^{2}=78.45, \mathrm{df}=2, p=<0.001$; Table 1).

\subsection{Control and volunteer survey groups}

We received completed surveys from 210 volunteers and 263 control group participants over three separate pairs of pre-season and post-season surveys. Some participants of both groups did not respond to certain questions within the survey so the sample sizes differ slightly. For the two pre/post-surveys in 2013 volunteers had an average $95.5 \%$ survey completion rate and the control group had an average survey completion rate of $73 \%$ (survey completion rate not tracked in 2012). We compared the ages, education level, income level, and housing density (rural, town/suburb, city) of the volunteer and control groups to ensure that they were similar. We found that both groups had a bimodal age distribution with peaks in the 20s and 60s, so we split the sample at age 40, and tested for differences between the age groups. There was no difference in the mean age of the younger group ( $t$-test, $t_{156}=1.24, p=0.22$ ), the older 
group had the statistically significant but biologically meaningless difference of 3.5 years in the mean age $\left(\operatorname{mean}_{\text {control }}=61.5\right.$, mean $_{\text {volunteer }}=58 ; t$-test, $\left.t_{313}=3.33, p=0.001\right)$. There was no difference in education level between the two groups (Chi-square, $\chi_{472}^{2}=3.65, p=0.16$ ), but the control group had more people who lived in rural areas (Chi-square, $\chi_{472}^{2}=9.70, p=0.008$ ), although the numbers living in town/suburban and city areas were the same as volunteers. The largest difference between groups was in income. The control group was concentrated in the middle income classes $(\$ 20-\$ 60 \mathrm{k}$ and $\$ 60-\$ 100 \mathrm{k})$ while our volunteer group was more evenly distributed through all income classes (Chi-square, $\chi_{438}^{2}=18.20, p=<0.001$ ).

\subsection{Volunteer experience}

The most commonly anticipated rewards for volunteering were similar to the actual rewards (Wilcoxon rank test, $N=198, p=0.76$ ). Before the project, volunteers were most excited about contributing to wildlife conservation $(22 \%, S E=5.2 \%)$ and capturing photographs of wildlife $(22 \%, S E=7.7 \%)$, while working outdoors in natural areas was a close third $(21 \% S E=7.3 \%)$. After the project, volunteers found that the most reward came from capturing photographs of wildlife $(25 \%, S E=3.5 \%)$, while working outdoors in natural areas $(22 \%, S E=2.5 \%)$ and contributing to wildlife conservation $(21 \%, S E=1.1 \%)$ were also common answers. The control group was similar, with most interested in capturing photographs of wildlife $(20 \%, S E=6.7 \%)$ and working outside $(22 \%, S E=4.1 \%)$, and we observed no difference in the pre and post-survey groups (Wilcoxon rank test, $N=74, p=0.79$ ). When volunteers described the best and worst part of the project in their own words, seeing wildlife and getting pictures were most often mentioned as the best part of the project (Figure 2), while time related issues, computer problems with uploading data, and various difficulties with setting out the cameras were all commonly mentioned as the worst part of volunteering.

\subsection{Wildlife knowledge}

Volunteers showed a significant but small improvement in their score in the wildlife ID quiz, with an average increase of $2.6 \%$ in the post test (Wilcoxon rank test, $n=186, p=0.005$, Cohen $\mathrm{d}=0.47$ ). The control group showed no change in wildlife knowledge scores in the before and after surveys $(t$-test, $t_{262}=0.193, p=0.85$, Cohen $\left.\mathrm{d}=0.015\right)$. Volunteers showed improvements in their knowledge of wildlife natural history after volunteering for both 2012 (paired- $t, t_{57}=-1.79, p=0.04$ ) and the combined 2013 surveys (paired- $\left.t, t_{124}=-5.72, p=<0.001\right)$. In 2012 the effect size was a $4.2 \%(n=58)$ increase, while the 2013 surveys showed a $12 \%(\mathrm{n}=125)$ increase. The control group showed no differences in the pre and post-survey scores in 2012 ( $t$-test, $\left.t_{110}=-1.25, p=0.11\right)$ or 2013 ( $t$-test, $\left.t_{173}=-1.53, p=0.07\right)$.

We invited half of our control and volunteer groups in the fall of 2013 to read entries about mammal natural history on the eMammal blog. Only $14.3 \%$ ( $n=6$ of 42) of the control group invited to use the blog reported reading the blog despite repeated invitations. For all 66 volunteers who were reminded about the blog, $50 \%(n=33)$ reported reading articles. We analyzed the difference in the pre and post natural history quiz scores of both groups and found that both the non-blog group ( $t$-test, $\left.t_{29}=2.40, p=0.01\right)$ and the blog group ( $t$-test, $t_{32}=3.58, p=<0.001$ ) showed a significant increase in their quiz scores. However the blog group mean increase was 2.04 times larger than the non-blog group (non-blog=0.833 vs. blog=1.70).

\subsection{Conservation attitudes}

The mean score of volunteers on the pre-test indices of all conservation attitudes were all above $80 \%$ of the maximum score ( $80 \%$ for wildlife attitudes, $96 \%$ for natural areas, and $88 \%$ conservation and economics). There was no difference in volunteer attitudes toward wildlife (paired- $t, t_{175}=1.93, p=0.97$ ), importance of natural areas (Wilcoxon rank test, $n=175, p=1.00$ ), or conservation economics (Wilcoxon rank test, $n=175, p=0.50$ ) after volunteering for the project. The control group had similarly high mean 
scores on conservation attitudes on the pre-test ( $79 \%$ for wildlife attitudes, $95 \%$ for natural areas, $89 \%$ conservation and economics). The control group in 2012 was a separate pre-test and post-test sample and in the 2013 seasons we surveyed the same individuals during pre and post-test surveys, so the groups are analyzed separately. There was no difference in the pre and post-test control group attitudes toward wildlife in 2012 ( $t$-test, $\mathrm{t}_{182}=-1.38, p=0.17$ ) or 2013 (paired- $t, t_{108}=-2.06, p=0.97$ ). There was also no difference in attitudes toward natural areas in 2012(Mann-Whitney, $\mathrm{z}_{182}=-1.53, p=0.13$ ) or 2013 (Wilcoxon rank test, $n=105, p=0.15$ ), or conservation and economics in 2012 (Mann-Whitney, $\mathrm{z}_{182}=1.09$, $p=0.27$ ) or 2013 (Wilcoxon rank test, $n=105, p=0.97$ ).

\subsection{Sharing information}

There was no difference in the number of volunteers that reported actively sharing wildlife information with their social network before and after volunteering. Half of the volunteers $(50 \%, n=90)$ reported sharing information about wildlife before volunteering and only slightly more than that after volunteering $(54 \%, \mathrm{n}=96)$ (McNemar's test, $\left.\chi_{180}^{2}=0.60, p=0.51\right)$. The control group also had no difference in the number of people reporting sharing wildlife information in the pre and post-surveys, with a little over half $(56 \%, \mathrm{n}=139)$ reporting sharing information in the pre-survey and just under half $(47 \%, \mathrm{n}=134)$ reporting sharing in the post-survey (McNemar's test, $\chi_{284}^{2}=2.36, p=0.12$ ).

However, after working for the project the type of information shared by volunteers changed. Before volunteering people shared wildlife information that ranged from identifying butterflies to polar bear conservation. After volunteering our citizen scientists were $84 \%$ more likely to discuss local mammals or local mammal conservation with their friends and family (McNemar's test, $\chi_{180}^{2}=4.74, p=0.04$, odds ratio=1.84). While there was no difference between the volunteer and control groups in the pre-survey (McNemar's test, $\chi_{438}^{2}=0.73, p=0.39$ ), post survey volunteers were 3.5 times more likely to talk to others about local mammals and local conservation than the control group (McNemar's test, $\chi_{447}^{2}=23.56$, $p=<0.001)$.

We found two top models explaining the variability in volunteers discussing local mammals or conservation (Table 2). The three variables that best explained variation in discussion were the number of predator sequences viewed, the age of the volunteer, and the volunteer's education level. Volunteers that viewed more predator sequences and older volunteers were more likely to discuss local mammals (Figure 3 ). There was also a trend that volunteers with professional or graduate degrees were more likely to talk about local mammals and natural history (Table 2).

\section{Discussion}

Citizen science projects have the potential to be powerful tools for conservation if they can affect participant's knowledge and attitudes while also gathering new scientific data (Bonney et al. 2009). In our citizen science project volunteers showed improvement in setting up camera deployments but not in wildlife identification. We were successful in changing volunteer's knowledge about mammals, but saw no change in attitudes toward conservation. Similar changes in project specific skills with no changes in attitudes have been seen in other short-term projects (Overdevest et al. 2004; Jordan et al. 2011; Crall et al. 2012). We also found that we influenced the type of information that volunteers shared with their social network, and finding that has implications for citizen science related to conservation.

\subsection{Improvement in volunteer skills and knowledge}

Volunteers showed a rapid improvement in deploying cameras with minimal feedback from project staff, and by the third month of the project approval rates were averaging about 99 percent. Volunteers received 
feedback about camera setup during training and a subset of volunteers received direct feedback in 2013. There was almost no feedback to volunteers about their errors in species identification because of the amount of data involved in the project, and volunteers showed little improvement in this skill. Other citizen science projects have found that feedback from staff to volunteers is important for both data quality and volunteer engagement (Berg et al. 2009; Jordan et al. 2011). Our findings confirm that feedback is important, and also show that in some situations volunteers may be capable of learning through self-directed feedback. Volunteers told project staff that they were able to improve the quality of their camera setup by comparing the pictures from their cameras to ideal setup examples from training. However, self-directed feedback was not possible with species identification because of the range in quality of photos (e.g. clear color pictures to blurry black and white images) and the variation among wildlife species.

Our review process was able to control the quality of the data from this project because we used camera photos as vouchers, or tangible proof of data collection. Based on the success of our review process, we echo the recommendation that citizen science programs use data vouchers when possible (Crall et al. 2011; Jordan et al. 2012b). While not applicable to all projects, there is a growing capacity to add vouchers in the form of recordings or photographs from mobile computing devices. Many plant, mammal, insect, and water quality projects could easily collect physical specimens or photographs as vouchers, which can be used to accurately assess data quality (Crall et al. 2011). Another advantage is that the act of gathering vouchers may help volunteers learn more about the specific taxon (Jordan et al. 2012a; Crall et al. 2012), as well as engage them in more hands-on activities in natural areas (Jordan et al. 2012a, 2012b). This direct experience has been linked to increased learning in informal situations about ecological processes and science (Dickinson et al. 2012). However, projects must be prepared to manage voucher specimens and have a data management plan to link field notes to voucher specimens. Digital vouchers also provide the opportunity to give volunteers rapid feedback. For example, eMammal volunteers could view updated information for their assigned cameras on their account dashboard on the eMammal website after entering data in the desktop app. We are also exploring ways to provide volunteers auto-generated emails with information about their identification accuracy via the eMammal website.

Volunteers did show a small but significant increase in knowledge about mammalian natural history during the course of their work. This knowledge was only peripherally related to the tasks of deploying cameras and identifying mammal photographs. Other citizen science projects have found that targeted educational materials help volunteers learn about ecology and natural history (Brossard et al. 2005), but we found that volunteers increased their knowledge of natural history without any targeted education. We also found that simply posting natural history information on a blog and inviting volunteers to read was sufficient to increase the amount that volunteers learned. This may have been related to the demographics of our volunteers, as most were from nature-based groups like Master Naturalist or Appalachian Trail Club. It was also likely related to the short time we followed volunteers (average of 3 months), and knowledge gains from longer projects are likely greater (e.g. Evans et al. 2005).

\subsection{Changes in how volunteers share information}

Our volunteers had strong positive attitudes toward wildlife and conservation at the start of the project, so it was not surprising that there was no further increase in their attitudes toward conservation after volunteering. It may also be unrealistic to expect to shift attitudes during part-time volunteer work over three to four months. Attitudes and beliefs are more complex constructs than knowledge, and are linked to a person's sense of identity and personal history (Brown et al. 2005). Volunteers are likely to self-select for projects that already match their attitudes and beliefs, and so volunteer activities will support existing 
attitudes. Projects that recruit from populations with more diverse attitudes or across broader age or education levels are more likely to reach people with divergent attitudes toward conservation.

We hypothesized that the excitement of obtaining wildlife photographs would encourage our volunteers to share wildlife information. Contrary to earlier research (Jordan et al. 2011) we found that the number of volunteers that shared information about wildlife with their networks did not change. Research has shown that this may be due to the connectedness in their personal networks (Travers \& Milgram 1969; Watts \& Strogatz 1998), and may reflect personality traits as much as subject area excitement (Baumeister \& Finkel 2010). Our project likely had no effect on the size of the volunteer's social network or their propensity to share information.

Although the number of volunteers who shared information about wildlife (aka "communicators") did not increase, these "communicators" shared different subject matter after volunteering for eMammal. We found that project volunteers formed an advocacy group for mammals and their conservation, as postproject "communicators" were $85 \%$ more likely to share information on local wildlife or local mammal conservation. This shift in sharing was enhanced by capturing photos of exciting wildlife, and volunteers who detected more predators on their cameras were more likely to share information about local mammals and conservation. Our results show that researchers should pay attention to what volunteer activities generate the most excitement since these activities have the highest potential for creating conservation advocates (see also Fore et al. 2001; Jordan et al. 2012b). Our findings show the potential of citizen science to spread support for conservation beyond the group of volunteers. More research is needed to discover how far information moves through social networks and whether this information is linked to behavior change (Watts \& Strogatz 1998; Girvan \& Newman 2002).

\subsection{Citizen science outside of the USA}

Community based monitoring and citizen science projects have also had success in other areas of the world, although planning and executing successful projects requires careful attention to the specific needs of the country or region where the project occurs (Gillies et al. In Press; Liebenberg et al. In press; Pintea et al. In press). Careful attention to local culture, key partnerships, establishing deliberative processes to guide projects, and providing adequate support for locals who are working on the project are critical for international projects (Berkes 2007). eMammal has also begun working internationally, and school groups that have partnered with local museums in both India and Mexico were excited and engaged with wildlife pictures captured during two eMammal projects that started in 2014 (Roland Kays, pers. comm.).

\subsection{Conclusions}

We were able to achieve education goals as part of an eMammal citizen science project that was focused mainly on scientific objectives. We hypothesize that the informal learning and the changes in shared information we observed was due to the exciting nature of camera trapping; discovering pictures of charismatic, hard-to-see mammals on the camera that one set is a very engaging activity. We join others in advocating the use of citizen science to address conservation problems (Cooper et al. 2007), especially when there is careful planning to achieve goals (Tulloch et al. 2013), and the use of vouchers to verify data quality (Crall et al. 2011). Citizen science can be an effective tool to connect people to the natural world and while simultaneously increasing support for conservation.

\section{Acknowledgements}

We thank all the eMammal volunteers for their hard work and dedication to this project. It would not have been possible without them. We also thank the state chapters of the Master Naturalists for their support of 
the project. We thank the staff of staff of several agencies for logistical support, including the National Parks, The Nature Conservancy, North Carolina Wildlife Resources Commission, Tennessee State Parks, Tennessee Division of Forestry, South Carolina State Parks, Virginia State Parks, Virginia Division of Game and Inland Fisheries, Western Virginia Water Authority, the Wintergreen Nature Foundation, Maryland State Parks, and Raleigh, Parks, Recreation and Cultural Resources. For help reviewing photographs, we thank N. Fuentes, S. Higdon, C. Bland, T. Perkins, L. Gatens, R. Owens, R. Gayle, C. Backman, K. Clark, J. Grimes, and J. Simkins. We thank M. Lubell and A. Crall for early help with study and survey design. Funding for the project was provided by an NSF Macrosystems Biology grant (grant no. 1232442). Logistical support and other funding was provided by the Smithsonian Institution, the North Carolina Museum of Natural Sciences, and North Carolina State University.

References

441

442

443

444

445

446

447

448

449

450

451

452

453

454

455

456

457

458

459

460

461

462

463

464

465

466

467

468

469

470

471

472

473

474

475

Arnold, T. W. 2010. Uninformative Parameters and Model Selection Using Akaike's Information Criterion. The Journal of Wildlife Management 74:1175-1178.

Baumeister, R. F., and E. J. Finkel. 2010. Advanced Social Psychology: The State of the Science. Oxford University Press.

Berg, H. A. V. D., S. L. Dann, and J. M. Dirkx. 2009. Motivations of Adults for Non-Formal Conservation Education and Volunteerism: Implications for Programming. Applied Environmental Education \& Communication 8:6-17.

Berkes, F. 2007. Community-Based Conservation in a Globalized World. Proceedings of the National Academy of Sciences of the United States of America 104:15188-15193.

Bonney, R., C. B. Cooper, J. Dickinson, S. Kelling, T. Phillips, K. V. Rosenberg, and J. Shirk. 2009. Citizen Science: A Developing Tool for Expanding Science Knowledge and Scientific Literacy. BioScience 59:977-984.

Brossard, D., B. Lewenstein, and R. Bonney. 2005. Scientific knowledge and attitude change: The impact of a citizen science project. International Journal of Science Education 27:1099-1121.

Brown, B. A., J. M. Reveles, and G. J. Kelly. 2005. Scientific literacy and discursive identity: A theoretical framework for understanding science learning. Science Education 89:779-802.

Burnham, K. P., and D. R. Anderson. 2002. Model selection and multi-model inference: A practical information-theoretic approach. Springer, New York, USA.

Campbell, D. T., and J. C. Stanley. 1963. Experimental and quasi-experimental designs for research. Houghton Mifflin Company, Boston, USA.

Cooper, C. B., J. Dickinson, T. Phillips, and R. Bonney. 2007. Citizen science as a tool for conservation in residential ecosystems. Ecology and Society 12:11.

Crall, A. W., R. Jordan, K. Holfelder, G. J. Newman, J. Graham, and D. M. Waller. 2012. The Impacts of an Invasive Species Citizen Science Training Program on Participant Attitudes, Behavior, and Science Literacy. Public Understanding of Science. Available from http://pus.sagepub.com/content/early/2012/04/05/0963662511434894 (accessed May 10, 2012).

Crall, A. W., G. J. Newman, T. J. Stohlgren, K. A. Holfelder, J. Graham, and D. M. Waller. 2011. Assessing citizen science data quality: an invasive species case study. Conservation Letters $4: 433-442$.

Dickinson, J. L., J. Shirk, D. Bonter, R. Bonney, R. L. Crain, J. Martin, T. Phillips, and K. Purcell. 2012. The current state of citizen science as a tool for ecological research and public engagement. Frontiers in Ecology and the Environment 10:291-297.

Dillman, D. A. 2011. Mail and Internet Surveys: The Tailored Design Method. John Wiley \& Sons. Eliasziw, M., and A. Donner. 1991. Application of the McNemar test to non-independent matched pair data. Statistics in Medicine 10:1981-1991. 
Evans, C., E. Abrams, R. Reitsma, K. Roux, L. Salmonsen, and P. Marra. 2005. The neighborhood nestwatch program: Participant outcomes of a citizen-science ecological research project. Conservation Biology: 19:589-594.

Falk, H.F., and L.D. Dierking. 2010. The 95 percent solution. American Scientist 98:486-493.

Fore, L. S., K. Paulsen, and K. O'Laughlin. 2001. Assessing the performance of volunteers in monitoring streams. Freshwater Biology 46:109-123.

Gillies, C. L., L. Ashcroft, M. R. Keatley, L. E. Chambers, K. Nga, A. Donnelly, R. Weatherill, R. Maitland, and J. Foster. In Press. ClimateWatch: a public engagement program tracking phenological events and species shifts in Australia. Biological Conservation.

Girvan, M., and M. E. J. Newman. 2002. Community structure in social and biological networks. Proceedings of the National Academy of Sciences 99:7821-7826.

Jordan, R., W. Brooks, D. Howe, and J. Ehrenfeld. 2012a. Evaluating the Performance of Volunteers in Mapping Invasive Plants in Public Conservation Lands. Environmental Management 49:425-434.

Jordan, R. C., H. L. Ballard, and T. B. Phillips. 2012b. Key issues and new approaches for evaluating citizen-science learning outcomes. Frontiers in Ecology and the Environment 10:307-309.

Jordan, R. C., S. A. Gray, D. V. Howe, W. R. Brooks, and J. G. Ehrenfeld. 2011. Knowledge Gain and Behavioral Change in Citizen-Science Programs. Conservation Biology 25:1148-1154.

Liebenberg, L., J. Steventon, K. Benadie, J. Minye, !Nate Brahman, H. K. Langwane, and Q. Xhukwe. In press. Towards an inclusive citizen science: From theory to practice. Biological Conservation.

Louv, R. 2008. Last child in the woods: Saving our children from nature-deficit disorder. Algonquin Books, Chapel Hill, USA.

McShea, W. J., T. Forrester, R. Costello, Z. He, and R. Kays. 2016. Volunteer-run cameras as distributed sensors for macrosystem mammal research. Landscape Ecology 31:55-66.

Miller, J.R. 2005. Biodiversity conservation and the extinction of experience. Trends in Ecology and Evolution 20:430-434.

Overdevest, C., C. H. Orr, and K. Stepenuck. 2004. Volunteer stream monitoring and local participation in natural resource issues. Human Ecology Review 11:177-185.

Pintea, L., T. Birch, T. Akugizibwe, and Y. Anokwa. In press. How innovative mobile and cloud technologies are empowering local communities and transforming chimpanzee conservation in Africa. Biological Conservation.

Purdy, K. G., and D. J. Decker. 1989. Applying Wildlife Values Information in Management: The Wildlife Attitudes and Values Scale. Wildlife Society Bulletin 17:494-500.

Travers, J., and S. Milgram. 1969. An Experimental Study of the Small World Problem. Sociometry 32:425-443.

Tulloch, A. I. T., H. P. Possingham, L. N. Joseph, J. Szabo, and T. G. Martin. 2013. Realising the full potential of citizen science monitoring programs. Biological Conservation 165:128-138.

Watts, D. J., and S. H. Strogatz. 1998. Collective dynamics of "small-world" networks. Nature 393:440442.

Weigel, L., and D. Metz. 2014. 2014 Conservation in the West. Pages 1-40. Poll. Colorado College. Available from https://www.coloradocollege.edu/dotAsset/ef6cac1a-da9d-4ede-a023eb138fd43ac8.pdf (accessed August 27, 2015).

Zaradic, P.A., O.R.W. Pergams, P. Kareiva. 2009. The impact of nature experience on willingness to support conservation. PLoS ONE 4(10): e7367. doi:10.1371/journal.pone.0007367 
Table 1

\begin{tabular}{lccr}
\hline \multirow{2}{*}{ Species or Deployment } & \multicolumn{3}{c}{ Error/Rejection Rate in Percent by Month (SE) } \\
\cline { 2 - 4 } & $33.3(5.6)$ & $19.9(6.3)$ & $21.2(7.5)$ \\
\hline Red Fox & $25.8(7.1)$ & $33.9(14.1)$ & $40.0(24.5)$ \\
Gray Fox & $10.5(1.7)$ & $11.9(1.8)$ & $14.8(2.5)$ \\
Eastern Gray Squirrel & $65.3(6.4)$ & $38.7(1.2)$ & $35.4(1.7)$ \\
Fox Squirrel & $55.1(4.1)$ & $58.7(3.9)$ & $65.4(3.7)$ \\
Unknown Species & & & $1.1(0.4)$ \\
& $15.1(2.5)$ & $5.6(1.3)$ &
\end{tabular}

520

521 The upper portion of the table shows the average error rates of identifications of visually similar species

522 pairs (red fox - gray fox, gray squirrel - fox squirrel) and species that were marked as unknown by

523 volunteers by the months of experience volunteers had with the project (e.g. all volunteers in their first

524 month are included in month 1 regardless of the calendar month that they started). The bottom of the table

525 shows the rejection rates of entire camera deployments for poor setup (e.g. too high) by months of

526 participation in the project. The standard error of each estimate is shown in parentheses. 
Table 2

\begin{tabular}{llllll}
\hline Top Models & Covariate & Odds Ratio & SE & $p$ value & 95\% Confidence Interval \\
\hline \multirow{2}{*}{ Model 1 } & Predator Detections & 1.42 & 0.20 & 0.01 & $1.07-1.87$ \\
& Age & 1.28 & 0.15 & 0.04 & $1.01-1.63$ \\
& & & & & \\
\multirow{2}{*}{ Model 2 } & Predator Detections & 1.40 & 0.20 & 0.02 & $1.06-1.85$ \\
& College Degree & 0.99 & 0.54 & 0.98 & $0.33-2.88$ \\
& Prof./Graduate Degree & 2.48 & 1.29 & 0.08 & $0.89-6.90$ \\
\hline
\end{tabular}

529 Results for all logistic regression models within $\Delta 2 \mathrm{AIC}_{\mathrm{c}}$ of the top model for sharing information about local mammals and conservation. Odds ratios greater than or less than 1 indicate an increase or decrease in the probability of sharing information respectively, and $95 \%$ confidence intervals and $p$ values show if the odds ratio is significantly different than one. Odds ratios for predator detections indicate the change in the probability of sharing information for every 50 predator detections seen, while age odds ratios indicate the change in probability of sharing information for every increase of 10 years of age. The odds ratios for college and professional/graduate degrees reflect the probability of volunteers with these degrees sharing information compared to volunteers with a high school education. 
546 Figure 1 - The study area of the eMammal project in the mid-Atlantic region of the USA. Each park is

547 represented by a point, cities are indicated with a star, and elevation is represented by shading with lighter 548 areas indicating higher elevation.

549 Figure 3 - The marginal change in the probability of sharing local mammals or conservation information 550 for different values of predator sequences seen and age of volunteers as predicted by the top logistic 551 regression model. The predicted probability is shown by the black line and $95 \%$ confidence intervals are 552 shown in gray. A typical volunteer saw from 60 to 70 sequences of predators and 30 volunteers saw more 553 than 100 predator sequences. 


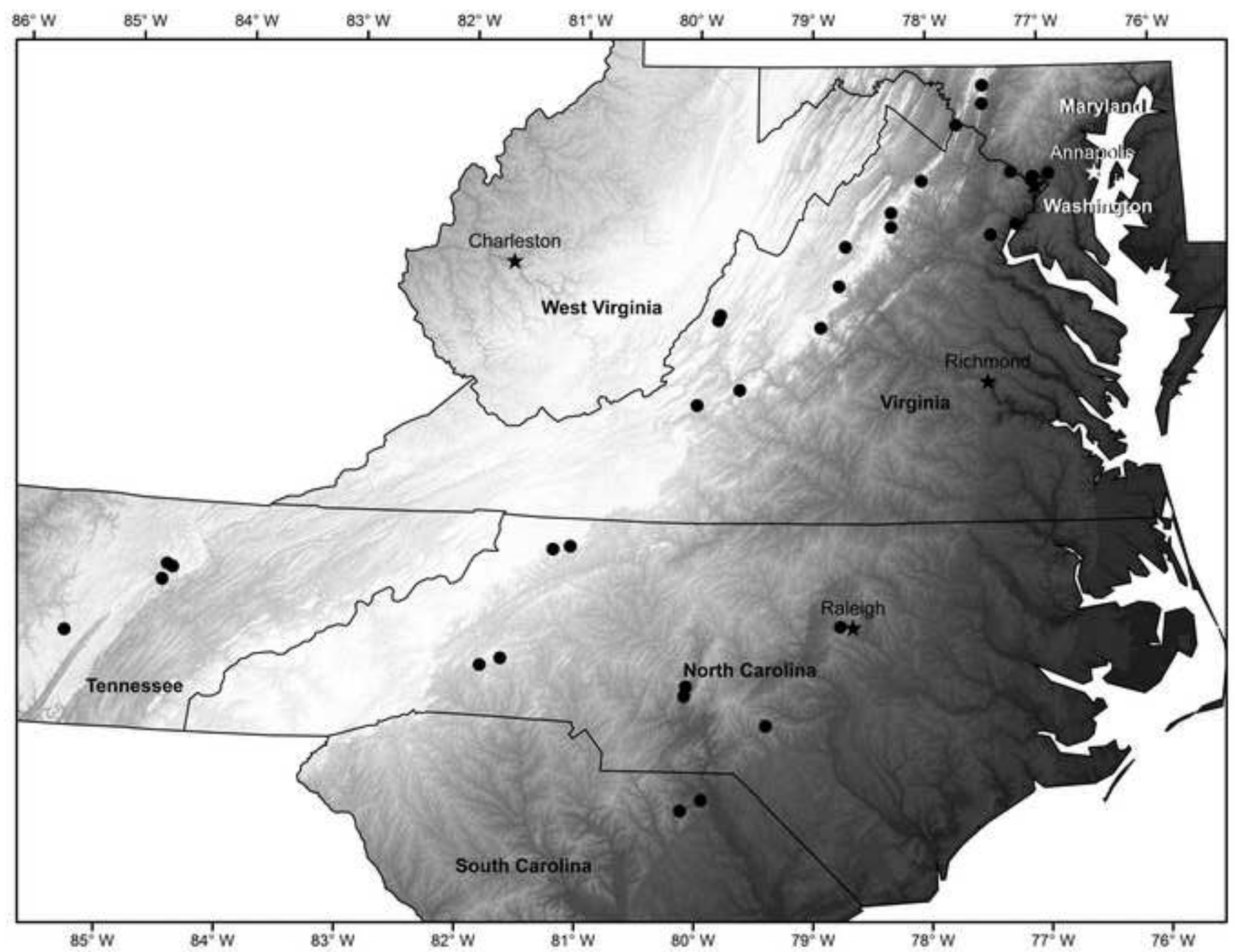




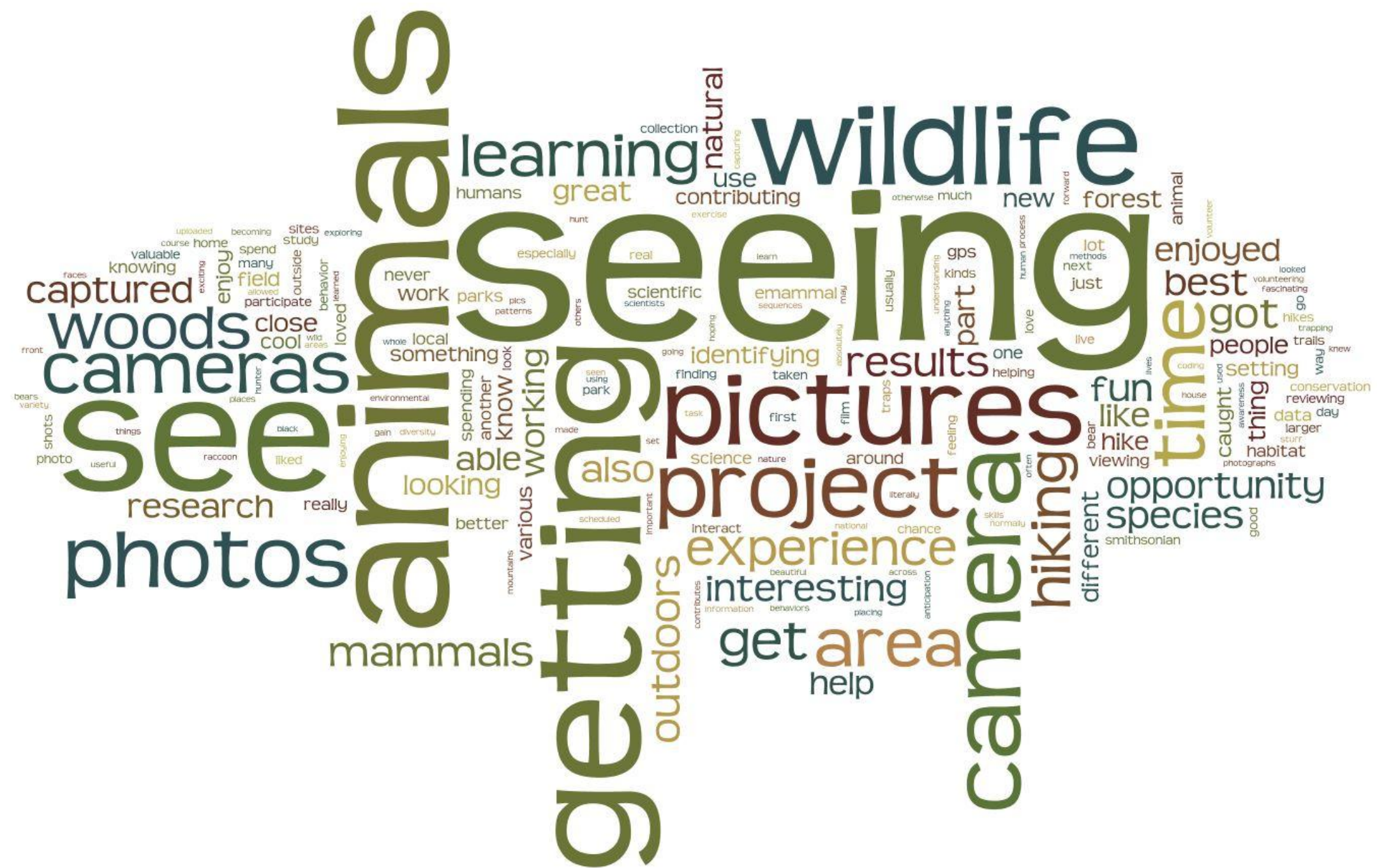

Figure 2 - The best part of volunteering for eMammal. A word cloud showing the responses of volunteers when asked to describe the best part of 556 volunteering for the eMammal project in their own words. The more a particular word occurs in the feedback the larger it appears in the word 557 cloud. 

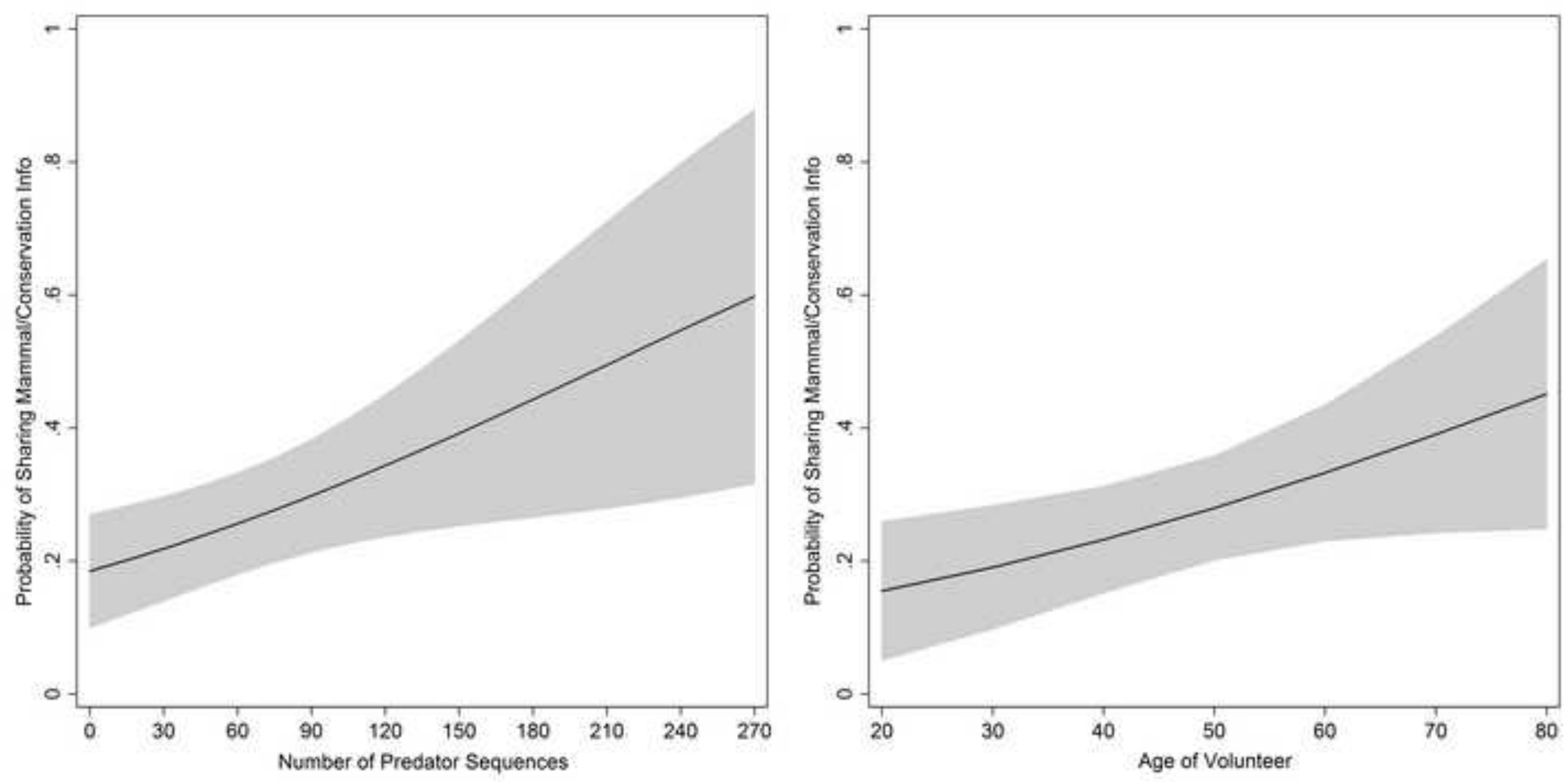